\title{
Utilizing Mobile-based Deep Learning Model for Managing Video in Knowledge Management System
}

\author{
https://doi.org/10.3991/ijim.v12i6.8563 \\ Harjanto Prabowo, Tjeng Wawan Cenggoro, Arif Budiarto, \\ Anzaludin Samsinga Perbangsa, Hery Harjono Muljo, Bens Pardamean \\ Bina Nusantara University, Jakarta, Indonesia \\ harprabowo@binus .edu
}

\begin{abstract}
Knowledge Management (KM) system is a core feature in facilitating intellectual growth in organization. However, there are numerous difficulties in maintaining a reliable KM system. One of the challenges is to manage knowledge materials in video format. A video file contains complex data that lead to the difficulties in managing them. Without an intelligent system, managing videos for $\mathrm{KM}$ requires a laborious effort. In this paper, an intelligent framework for KM system, embedded with deep learning model, is proposed. The use of the deep learning model alleviates the heavy burden of video materials management in KM system. To enhance the agility of the system, mobile-based deep learning model is utilized in the framework.
\end{abstract}

Keywords-deep learning, knowledge management, video classification, mobile-based

\section{Introduction}

As a discipline, Knowledge Management (KM) provides a framework to efficiently manage and make use of knowledge within organization. KM focuses on managing knowledge contents so that they can deliver useful information to organization. However, knowledge for organizations or individuals cannot be managed with a single technology, but requires technology equipped with Artificial Intelligence (AI) facilities to run it and to achieve maximum results [1]. For instance, managing knowledge in video format require extraction of underlying information in the video itself. By automating the extraction process with AI, the needs of human to infer the information in videos can be reduced, which in turns speed up processes of managing knowledge in video format.

To further accelerate processes in KM, the use of mobile technologies can also be integrated within the framework. Infusing mobile technologies in a KM system enables knowledge workers to interact with the system without constrained by time and location [2]. This leads to a better agility in the overall KM framework.

Considering the benefits of both AI and mobile technologies in KM framework, it is promising to have a clear strategy for integrating them within the framework. This paper introduces a design that incorporates $\mathrm{AI}$ and mobile technology in KM framework, 
particularly in managing video content. The type of AI used in the design is deep learning [3], which is the state-of-the-art technique for video analysis [4].

\section{Knowledge management}

Knowledge management $(\mathrm{KM})$ is a subject area which has potential to open new paradigm in organization. The implementation of $\mathrm{KM}$ is relevant in industrial environment due to its capability in increasing organization performance, so that it can be considered as a highly-valued asset for management [5]. KM is an essential factor for organization strength and sustainability [6]. KM also provides organization with strategy to increase profit, competitiveness, and capacity [7][8].

KM allows organization to exploit its human intellectual asset to improve competitive advantage. KM also plays a critical role in immersing new business process design, mapping cultivated knowledge, and utilizing business intelligence to fulfil organization needs. In addition, KM is also aligned with the goals of capturing, sharing and reusing knowledge within an organisation or among organisations [9].

The benefit of KM is not only limited to organization in high-technology industry, but also to organization mainly engaged in economy, education, banking, telecommunication, manufacture, and public sector [10][11]. Therefore, KM can be considered as the main driving force of organization performance [12]. More interestingly, KM can also be considered as a vital resource for organization viability and prosperity [13][14].

\section{Deep learning for video classification}

The growth of Internet use nowadays has generated a massive amount of data in the form of multimedia content, especially in video content. This trend has motivated the development of AI techniques to extract useful information from video content [15]. One of the most practical application of $\mathrm{AI}$ in information extraction from video is video classification. Video classification is a problem where the goal is to categorize videos into separate labels which semantically have meanings to human. This particular application has been deployed in managing a massive amount of video data [16].

With the recent successes of deep learning in image analysis [17][18][19][20], the latest advances in video classification also intensively use deep learning techniques. The deep learning techniques for video classification can generally be grouped into three big categories: CNN-LSTM [21][22], 3D CNN [16][23][24], and Two Stream Network [25][26]. The first category, CNN-LSTM technique, leverages combination of Long Short Term Memory (LSTM) [27][28], which works well in temporal data analysis, and Convolutional Neural Network (CNN) [29], which excels in handling image data. The second category, 3D CNN, treats the temporal dimension of videos as spatial dimension, and incorporates it as additional dimension in CNN, which normally operates using 2D image data. The last category, Two Stream Network, use two 3D $\mathrm{CNN}$ with different input, and then fuse the results for final classification. The two inputs used are frames from video and its optical flow [30]. The addition of optical flow 
input gives temporal information to the vanilla 3D CNN, thus tends to work better than normal 3D CNN.

\section{$4 \quad$ Related works}

The implementation of artificial intelligence (AI) to support knowledge management (KM) has been explored by scientists for more than a decade. One of the main reasons behind this relationship is because both of these computer science's subsets employee the same concepts called knowledge base and ontology [31]. Knowledge base is the main component of $\mathrm{KM}$ that contains all information or knowledge shared in a community or organization. Akin to that concept, AI also has knowledge base as the main data source that can enrich the model to solve particular problem. Whilst, ontology is an important part for $\mathrm{KM}$ and $\mathrm{AI}$ to understand every entity from the knowledge base. Without an ontology, users of knowledge management system (KMS) will find it difficult to understand some parts of the knowledge that relatively new for them. Ontology in AI is used differently. It is used to ensure a computer to understand any kind of input data with in a such way that it can have the same understanding with human.

In 2001, Liebowitz has already noticed that AI was the building block in KM development and advancement [32]. This issue had been there because of the fact that knowledge management system was no more than an information or document management product. The absence of $\mathrm{AI}$ in this so-called $\mathrm{KM}$, at that time, was the main reason why there were a lot of doubts for managerial levels to adopt KM in their organizations. Liebowitz clearly states that AI supports KM in every aspects ranging from knowledge sharing, individual to collective and organizational knowledge transformation, and end up in the reincarnating from organizations into knowledge organizations. Furthermore, Liebowitz also mentioned two important parts of AI that can be applied in KM. The first one is the knowledge acquisition technique that can be applied in KM to build knowledge repository. The second one is Intelligent Agent technique that can be used to store and retrieval method in KM system [33].

Another connection between KM and AI lies on the concept of intelligent data warehouse development, powered by AI, to make data store and retrieval process become effortless [34]. One of the basic pillars of KM is to digitize every knowledge within organization so that it can be shared and reused by other members of the organization. Nemati et al proposed a new extension of data warehouse model powered by AI called knowledge warehouse. Their proposed concept of data warehouse was not only focus on the knowledge capturing and coding. More important thing, this data warehouse can be an effective tool for users to store, manage and retrieve every knowledge they need. These processes including knowledge filtering technique that use AI to analyze natural text [35]. In this case AI can provide benefits in every layer of knowledge management system from tacit to tacit knowledge sharing to explicit to tacit knowledge conversion. Additionally, this knowledge warehouse will not stop just to convert knowledges, but also will end up in decision making process. Additionally, in their research project, Nemati et al also highlighted the usefulness of AI to manage and 
process all knowledges so that it can enrich users in the decision making process using one specifically AI application called expert system [32].

AI has been applied to support KM in several domains. One of them is knowledge management for health or biomedical domain. One main reason why knowledge management is very important in this domain is because the massive amount of data involved. Holzinger and Jurisica (2014) highlighted this important point by stating that one big challenge in biomedical research is the data overload [36]. They proposed an effective solution as the knowledge discovery and data mining in their research. This interactive solution help users to manage large and unstructured data as well as to optimize the overall pipeline and workflow. They implemented a combination approach between Human Computer Interaction and Knowledge Discovery \& Data Mining (HCI-KDD) in building their solution [37].

Education institutions also experience a similar thing with biomedical researchers. They have to deal with large amount data related to the knowledge itself and also other data such as students, lecturers, and staffs. A comprehensive study has been introduced by Lara et al 2014 to investigate the best approach to build knowledge discovery system for Open University of Madrid, UDIMA [38]. Their main consideration in building the solution was the ability of the students to reconcile between their desire to study and their job and family responsibilities. E-learning approach was believed to be the best answer for that problem. Consequently, the complexity of data and information in this particularly case become more complicated. Began with that problem, Lara et al developed an effective tool to help lecturers in transferring the knowledges. They used a basic Virtual Learning Environment (VLE) called Moodle [39]. Based on this VLE they developed a knowledge discovery and data mining model that can be applied in Moodle platform. Historical and participative data were the basis of analysis in the proposed model. At the end, the model can be used to predict students' grade, detect students' behaviour, advice lecturers with all information about the class, and build a tool for student modeling.

The research of $\mathrm{AI}$ integration to $\mathrm{KM}$ so far utilized $\mathrm{AI}$ techniques which fall to either expert system or data mining category. While deep learning has been known as state-of-the-art technique in almost all AI tasks, there is no research that incorporates deep learning in $\mathrm{KM}$ to the best of our knowledge.

\section{$5 \quad$ Research methodology}

This study used unified modeling language (UML) as a standard set of notations for system modeling. UML provides Use case diagrams, activity diagrams, and class diagrams that can be used to model structure and behavior systems. The use case diagram is used to determine user role group and what features will be used in the system. The activity diagram describes the workflow of user and system. And finally the class diagram will be used to describes the class of a collection of similar objects and relationships between classes.

The proposed KM system framework harness the most advanced method in artificial intelligence called deep learning. This method has been proved to be able to create 
robust system that invloves videos as the data input [16][22][25][40]. For the sake of mobility, this deep learning method is implemented in mobile technology by using a light CNN model for mobile called as MobileNet [41]. The architecture of MobileNet can be conveniently adjusted to fit in mobile device, yet has a comparable performance to VGGNet [42] and AlexNet [17], two of the most highly performed CNN models for computer vision.

MobileNet utilizes the concept of Depthwise Separable Convolution (DSC) in its architecture. This concept is originally developed in 2014 by Sifre [43] and formulated for use in CNN by Chollet in 2016 [44]. DSC works by using parallel modules of stacked convolutional layers, which are pooled in the end to form a single output channel. MobileNet introduce a parameter $\alpha$ which controls the number of parallel modules in DSC. By controlling $\alpha$, MobileNet can be squeezed to fit in mobile hardware by sacrificing only a little accuracy.

For the development and deployment of MobileNet model, Tensorflow framework [45] is utilized. Specifically, the version of Tensorflow utilized is Tensorflow Lite, the version optimized for mobile. The use of Tensorflow Lite allows a dramatic acceleration to develop Deep Learning based application in mobile, because this framework can be coded and tested directly in a mobile device.

\section{Results and discussion}

The functional requirement of Mobile KMS is presented in the use case diagram as shown in Figure 1.

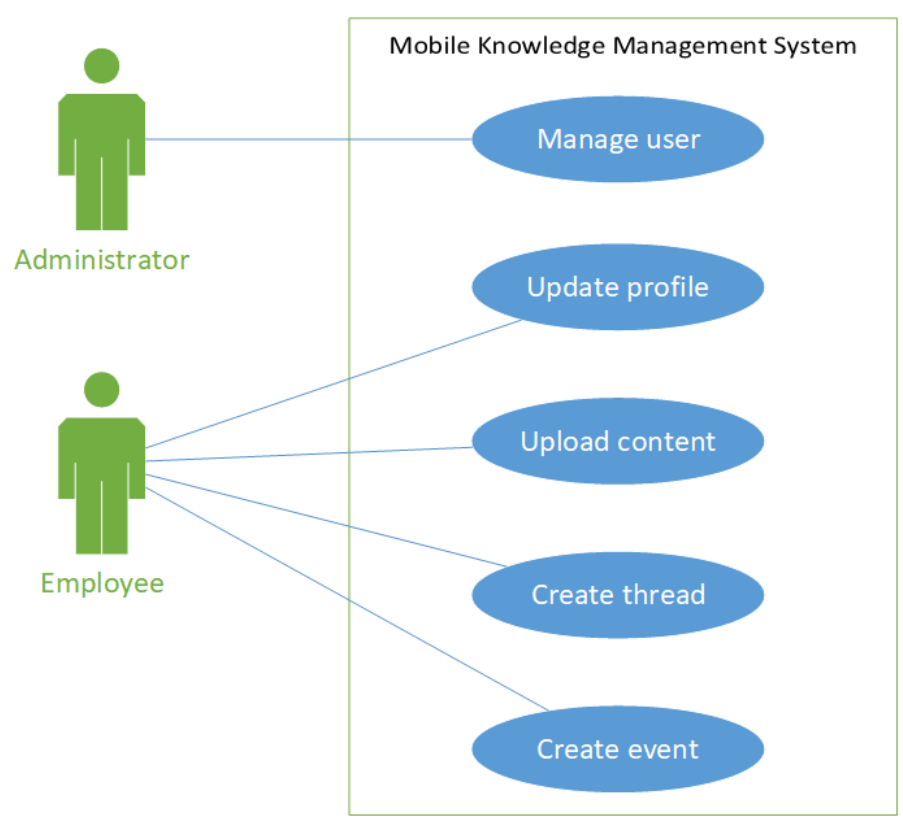

Fig. 1. Use Case Diagram 
The administrator is responsible for managing user data and granting the appropriate user's access role in the system. Administrators are also responsible for updating application-related information to users. Employees as an expert can share their knowledge by uploading learning materials including video contents. Knowledge dissemination can also be done by creating threads in the discussion forum feature. Based on the use case diagram, an activity diagram was created in Figure 2 that describing the mobile KMS workflow.

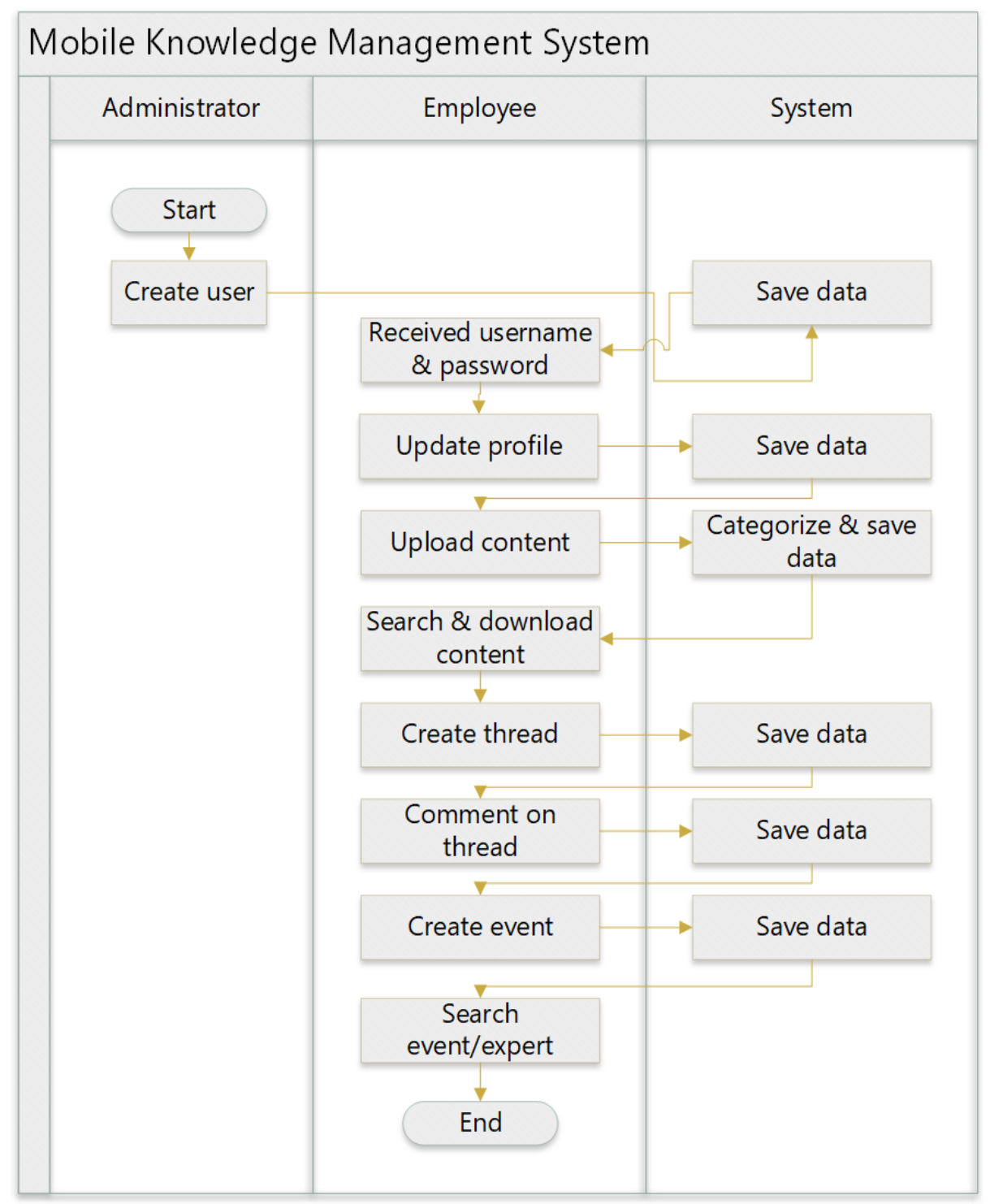

Fig. 2. Activity Diagram 
To be able to use the system, employees must be enrolled first by the administrator. When employees are able to access entry into the system, can manage the profile and change the password for security. Employees as an expert can upload learning materials, including videos that will automatically be categorized by the system using deep learning. Employees who need knowledge can search and download content based on categories that have been compiled by the system with deep learning. After learning the content, employees can also improve and share knowledge through discussion forums. Employees can also share information about an activity related to certain knowledge such as training, workshops, and seminars through event features. The relationships between classes are shown in Figure 3.

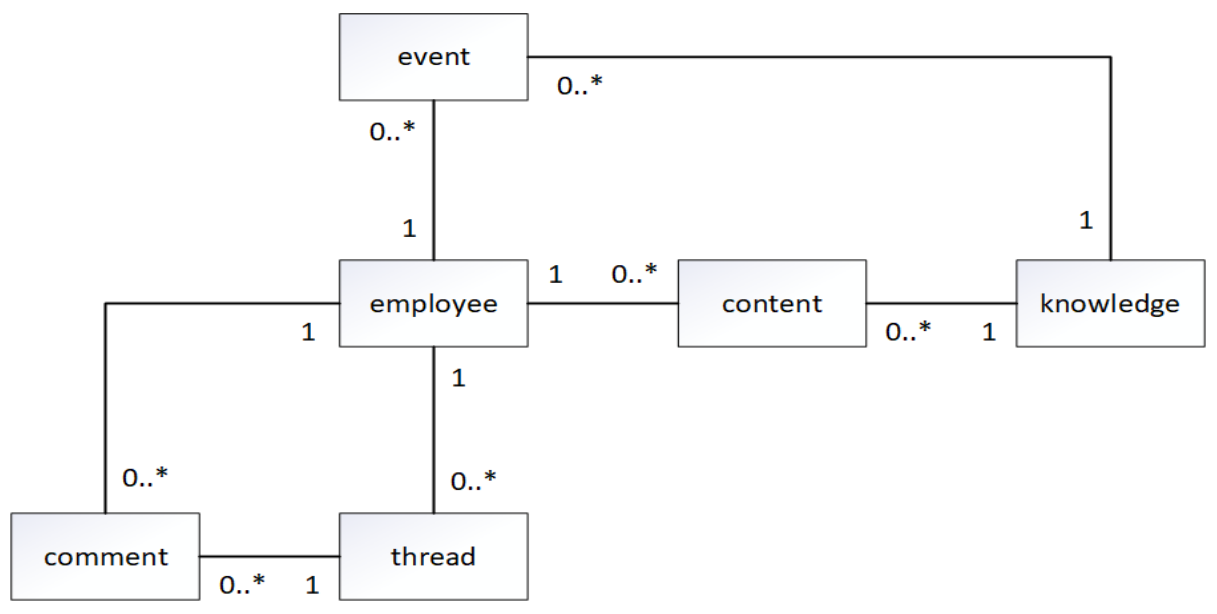

Fig. 3. Class Diagram

The class diagram consists of six classes, namely employee, knowledge, thread, event, content, and comment. Attributes in the employee class are biodata, login access, and role in the system. Class knowledge contains attribute categories and inheritance attribute to the parent knowledge. The thread class contains the topic attribute in the discussion forum. Content contains shared knowledge file attribute. And the class comment contains the attribute of the question/opinion/suggestion in the discussion forum.

The categorize and save data activity in Figure 2 is specifically targeted for video data. This activity is where the deep learning algorithm for video classification is placed. By using deep learning, the categorize and save data activity can be automated and thus increase the efficiency of human labors in the whole KM system. Specifically, the deep learning algorithm used is 3D CNN [23] with raw RGB input. The 3D CNN architecture used is Inflated 3D (I3D) CNN model [4] pretrained with ImageNet dataset [46]. Different from the original I3D CNN, the CNN structure used in the system is MobileNet, because the system will be installed in mobile device. The architecture of the proposed I3D MobileNet model is described in Table 1. The notation of conv in 
Type column in Table 1 means convolutional layer, dw means DSC layer, and dw in Filter Shape column means number of parallel convolutional layers for DSC layer.

The strategy of using raw RGB input is chosen because it is the most efficient strategy to be implemented in mobile device. For comparison, the state-of-the-art video analysis strategy use auxiliary optical flow input to enhance its accuracy [4]. The additional optical flow input introduces extra computations for calculating the optical flow, while can only increase the accuracy by $2.4 \%$ [4]. The other common video analysis strategy which utilizes LSTM layer is also not an appropriate option. Computing both CNN and LSTM as one model is an intense operation which should be avoided for implementation in mobile device. Moreover, the state-of-the-art technique using LSTM can only achieve performance lower than the chosen strategy. Specifically, the accuracy is $1 \%$ lower.

Table 1. Proposed I3D MobileNet Architecture

\begin{tabular}{|c|c|c|c|c|}
\hline \multicolumn{2}{|c|}{\begin{tabular}{|r|} 
Type \\
\end{tabular}} & Stride & Filter Shape & Input Size \\
\hline \multicolumn{2}{|c|}{ Conv } & 2 & $3 \times 3 \times 3 \times 3 \times 32$ & $224 \times 224 \times 64 \times 3$ \\
\hline \multicolumn{2}{|c|}{ Conv dw } & 1 & $3 \times 3 \times 3 \times 32 \mathrm{dw}$ & $112 \times 112 \times 32 \times 32$ \\
\hline \multicolumn{2}{|c|}{ Conv } & 1 & $1 \times 1 \times 1 \times 32 \times 64$ & $112 \times 112 \times 32 \times 32$ \\
\hline \multicolumn{2}{|c|}{ Conv dw } & 2 & $3 \times 3 \times 3 \times 64 d w$ & $112 \times 112 \times 32 \times 64$ \\
\hline \multicolumn{2}{|c|}{ Conv } & 1 & $1 \times 1 \times 1 \times 64 \times 128$ & $56 \times 56 \times 16 \times 64$ \\
\hline \multicolumn{2}{|c|}{ Conv dw } & 1 & $3 \times 3 \times 3 \times 128 d w$ & $56 \times 56 \times 16 \times 128$ \\
\hline \multicolumn{2}{|c|}{ Conv } & 1 & $1 \times 1 \times 1 \times 128 \times 128$ & $56 \times 56 \times 16 \times 128$ \\
\hline \multicolumn{2}{|c|}{ Conv dw } & 2 & $3 \times 3 \times 3 \times 128 \mathrm{dw}$ & $56 \times 56 \times 16 \times 128$ \\
\hline \multicolumn{2}{|c|}{ Conv } & 1 & $1 \times 1 \times 1 \times 128 \times 256$ & $28 \times 28 \times 8 \times 128$ \\
\hline \multicolumn{2}{|c|}{ Conv dw } & 1 & $3 \times 3 \times 3 \times 256 \mathrm{dw}$ & $28 \times 28 \times 8 \times 256$ \\
\hline \multicolumn{2}{|c|}{ Conv } & 1 & $1 \times 1 \times 1 \times 256 \times 256$ & $28 \times 28 \times 8 \times 256$ \\
\hline \multicolumn{2}{|c|}{ Conv dw } & 2 & $3 \times 3 \times 3 \times 256 \mathrm{dw}$ & $28 \times 28 \times 8 \times 256$ \\
\hline \multicolumn{2}{|c|}{ Conv } & 1 & $1 \times 1 \times 1 \times 256 \times 512$ & $14 \times 14 \times 4 \times 256$ \\
\hline \multirow{2}{*}{$5 x$} & Conv dw & 1 & $3 \times 3 \times 3 \times 512 \mathrm{dw}$ & $14 \times 14 \times 4 \times 512$ \\
\hline & Conv & 1 & $1 \times 1 \times 1 \times 512 \times 512$ & $14 \times 14 \times 4 \times 512$ \\
\hline \multicolumn{2}{|c|}{ Conv dw } & 2 & $3 \times 3 \times 3 \times 512 \mathrm{dw}$ & $14 \times 14 \times 4 \times 512$ \\
\hline \multicolumn{2}{|c|}{ Conv } & 1 & $1 \times 1 \times 1 \times 512 \times 1024$ & $7 \times 7 \times 2 \times 512$ \\
\hline \multicolumn{2}{|c|}{ Conv dw } & 2 & $3 \times 3 \times 3 \times 1024 \mathrm{dw}$ & $7 \times 7 \times 2 \times 1024$ \\
\hline \multicolumn{2}{|c|}{ Conv } & 1 & $1 \times 1 \times 1 \times 1024 \times 1024$ & $7 \times 7 \times 1 \times 1024$ \\
\hline \multicolumn{2}{|c|}{ Average Pooling } & 1 & Pool 7×7x1 & $7 \times 7 \times 1 \times 1024$ \\
\hline \multicolumn{2}{|c|}{ Fully Connected } & 1 & $1024 \times 1000$ & $1 \times 1 \times 1024$ \\
\hline \multicolumn{2}{|c|}{ Softmax } & 1 & Classifier & $1 \times 1 \times 1000$ \\
\hline
\end{tabular}

\section{Conclusion}

This paper demonstrates the integration strategy for deep learning based video classification system into KM system, in particular for handling video content. This integration enables one activity in the proposed KM system to be automated, which 
alleviates ineffective use of human resource in the whole system. In regards to the utilization of mobile technology in the KM system, the deep learning based video classification model proposed in this paper is I3D MobileNet. The characteristic of this model is applicable to mobile hardware constraint yet powerful enough to be used for video content management in KM system. The future works following this paper should provide the implementation results of the proposed integration strategy.

\section{Acknowledgement}

This study was supported by Artificial Intelligence Research \& Development Center of Bina Nusantara University.

\section{References}

[1] Birzniece, I. (2011). Artificial Intelligence in Knowledge Management: Overview and Trends. Scientific Journal of Riga Technical University, 6, 5-11. https://doi.org/10.2478/ v10143-011-0001-X

[2] Zhang, Z. (Justin), \& Jasimuddin, S. M. (2015). A model-based analysis for mobile knowledge management in organizations. Journal of Management Analytics, 2(1), 35-52. https://doi.org/10.1080/23270012.2015.1012232

[3] LeCun, Y., Bengio, Y., \& Hinton, G. (2015). Deep learning. Nature, 521(7553), 436-444. https://doi.org/10.1038/nature14539

[4] Carreira, J., \& Zisserman, A. (2017). Quo Vadis, Action Recognition? A New Model and the Kinetics Dataset. arXiv Prepr. https://doi.org/10.1109/CVPR.2017.502

[5] Spender, J. C. (2008). Organisational learning and knowledge management: whence and wither?. Management Learning, 39(2), 158-176. https://doi.org/10.1177/1350507 607087582

[6] Riege, A. (2007). Actions to overcome knowledge transfer barriers in MNCs. Journal of Knowledge Management, 11(1), 48-67. https://doi.org/10.1108/13673270710728231

[7] Chua, A. Y. K. (2009). The dark side of successful knowledge management initiatives. Journal of Knowledge Management, 13(4), 32-40. https://doi.org/10.1108/13673270910971806

[8] Jeon, S., Kim, Y., \& Koh, J. (2011). An integrative model for knowledge sharing in communities-of-practice. Journal of Knowledge Management, 15(2), 251-269. https://doi.org/10.1108/13673271111119682

[9] Tsui, E., Garner, B. J., \& Staab, S. (2000). The Role of Artificial Intelligence in Knowledge Management. Editorial Note, 13, 235-239. https://doi.org/10.1016/S0950-7051(00)00093-9

[10] Teng, J. T. C. \& Song, S. (2011). An exploratory examination of knowledge-sharing behaviours: solicited and voluntary. Journal of Knowledge Management, 15(1), 104-117. https://doi.org/10.1108/13673271111108729

[11] Zack, M. H. (2003). Rethinking the knowledge-based organization. Sloan Management Review, 44(4), 67-71.

[12] Bosua, R., \& Venkitachalam, K. (2013). Aligning strategies and processes in knowledge management: a framework. Journal of Knowledge Management, 17(3), 331-346. https://doi.org/10.1108/JKM-10-2012-0323 
[13] Teece, D. F., Pisano, G. \& Shuen, A. (1997). Dynamic capabilities and strategic management. Strategic Management Journal, 18(7), 509-533. https://doi.org/10.1002/(SICI)10970266(199708)18:7<509::AID-SMJ882>3.0.CO;2-Z

[14] Kamhawi, E. M. (2012). Knowledge management fishbone: A standard framework of organisational enablers. Journal of Knowledge Management, 16(5), 808-828. https://doi.org/10.1108/13673271211262826

[15] Wu, Z., Yao, T., Fu, Y., \& Jiang, Y. G. (2016). Deep Learning for Video Classification and Captioning. arXiv preprintarXiv:1609.06782.

[16] Karpathy, A., \& Leung, T. (2014). Large-scale Video Classification with Convolutional Neural Networks. Proc. 2014 IEEE Conf. Comput. Vis. Pattern Recognit., 1725-1732. https://doi.org/10.1109/CVPR.2014.223

[17] Krizhevsky, A., \& Hinton, G. E. (2012). ImageNet Classification with Deep Convolutional Neural Networks. In Advances in Neural Information Processing Systems.

[18] Ren, S., He, K., Girshick, R., \& Sun, J. (2017). Faster R-CNN: Towards real-time object detection with region proposal networks. IEEE Transactions on Pattern Analysis and Machine Intelligence, 39(6), 91-99. https://doi.org/10.1109/TPAMI.2016.2577031

[19] He, K., Gkioxari, G., Dollar, P., \& Girshick, R. (2017). Mask R-CNN. In 2017 IEEE International Conference on Computer Vision (ICCV) (pp. 2980-2988). IEEE. https://doi.org/10.1109/ICCV.2017.322

[20] He, K., Zhang, X., Ren, S., \& Sun, J. (2015). Deep Residual Learning for Image Recognition. Arxiv.Org, 7(3), 171-180. https://doi.org/10.3389/fpsyg.2013.00124

[21] Donahue, J., Hendricks, L. A., Rohrbach, M., Venugopalan, S., Guadarrama, S., Saenko, K., \& Darrell, T. (2017). Long-Term Recurrent Convolutional Networks for Visual Recognition and Description. IEEE Transactions on Pattern Analysis and Machine Intelligence, 39(4), 677-691. https://doi.org/10.1109/TPAMI.2016.2599174

[22] Ng, J. Y. H., Hausknecht, M., Vijayanarasimhan, S., Vinyals, O., Monga, R., \& Toderici, G. (2015). Beyond short snippets: Deep networks for video classification. In Pro-ceedings of the IEEE conference on computer vision and pattern recognition (pp. 4694-4702).

[23] Ji, S., Xu, W., Yang, M., \& Yu, K. (2013). 3D Convolutional Neural Networks for Human Action Recognition, IEEE Trans. Pattern Anal. Mach. Intell., 35(1), 221-231. https://doi.org/10.1109/TPAMI.2012.59

[24] Taylor, G. W., Fergus, R., LeCun, Y., \& Bregler, C. (2010). Convolutional learning of spatio-temporal features. Lecture Notes in Computer Science (Including Subseries Lecture Notes in Artificial Intelligence and Lecture Notes in Bioinformatics), 6316 LNCS(PART 6), 140-153. https://doi.org/10.1007/978-3-642-15567-3_11

[25] Simonyan, K., \& Zisserman, A. (2014). Two-stream convolutional networks for action recognition in videos. Advances in Neural Information Processing Systems, 568-576.

[26] Feichtenhofer, C., Pinz, A., \& Zisserman, A. (2016). Convolutional two-stream network fusion for video action recognition. In Proceedings of the IEEE Conference on Computer Vision and Pattern Recognition (pp. 1933-1941). https://doi.org/10.1109/CVPR.2016.213

[27] Hochreiter, S., \& Schmidhuber, J. (1997). Long Short-Term Memory. Neural Computation, 9(8), 1735-1780. https://doi.org/10.1162/neco.1997.9.8.1735

[28] Gers, F. A., Cummins, F., Schmidhuber, J., \& Cummins, F. (2000). Learning to forget: continual prediction with LSTM. Neural Comput., 12(10), 2451-2471. https://doi.org/10.1162/ 089976600300015015

[29] LeCun, Y., Boser, B., Denker, J. S., Henderson, D., Howard, R. E., Hubbard, W., \& Jackel, L. D. (1989). Backpropagation Applied to Handwritten Zip Code Recognition. Neural Computation. https://doi.org/10.1162/neco.1989.1.4.541 
[30] Brox, T., Bruhn, A., Papenberg, N., \& Weickert, J. (2004). High Accuracy Optical Flow Estimation Based on a Theory for Warping. In Pajdla T., Matas J. (eds) Computer Vision ECCV 2004. ECCV 2004. Lecture Notes in Computer Science, vol 3024 (pp. 25-36). https://doi.org/10.1007/978-3-540-24673-2_3

[31] O'Leary, D. E. (1998). Using AI in knowledge management: Knowledge bases and ontologies. IEEE Intelligent Systems and their Applications, 13(3), 34-39. https://doi.org/10.1109/ $\underline{5254.683180}$

[32] Liebowitz, J. (2001). Knowledge management and its link to artificial intelligence. Expert systems with applications, 20(1), 1-6. https://doi.org/10.1016/S0957-4174(00)00044-0

[33] Bradshaw, J. M., Carpenter, R., Cranfill, R., Jeffers, R., Poblete, L., Robinson, T., ... \& Sullivan, K. (1997). Roles for agent technology in knowledge management: examples from applications in aerospace and medicine. AAAI Technical Report SS-97-01, 9-16.

[34] Nemati, H. R., Steiger, D. M., Iyer, L. S., \& Herschel, R. T. (2002). Knowledge warehouse: an architectural integration of knowledge management, decision support, artificial intelligence and data warehousing. Decision Support Systems, 33(2), 143-161.S https://doi.org/10.1016/S0167-9236(01)00141-5

[35] Li, Y., Zhang, C., \& Swan, J. R. (2000). An information filtering model on the Web and its application in JobAgent. Knowledge-Based Systems, 13(5), 285-296. https://doi.org/10.1016/S0950-7051(00)00088-5

[36] Holzinger, A., \& Jurisica, I. (2014). Knowledge discovery and data mining in biomedical informatics: The future is in integrative, interactive machine learning solutions. In Interactive knowledge discovery and data mining in biomedical informatics (pp. 1-18). Springer Berlin Heidelberg. https://doi.org/10.1007/978-3-662-43968-5 1

[37] Holzinger, A. (2013, September). Human-Computer Interaction and Knowledge Discovery (HCI-KDD): What is the benefit of bringing those two fields to work together?. In International Conference on Availability, Reliability, and Security (pp. 319-328). Springer, Berlin, Heidelberg. https://doi.org/10.1007/978-3-642-40511-2 22

[38] Lara, J. A., Lizcano, D., Martínez, M. A., Pazos, J., \& Riera, T. (2014). A system for knowledge discovery in e-learning environments within the European Higher Education Area-Application to student data from Open University of Madrid, UDIMA. Computers \& Education, 72, 23-36. https://doi.org/10.1016/j.compedu.2013.10.009

[39] Dougiamas, M., \& Taylor, P. (2003). Moodle: Using learning communities to create an open source course management system.

[40] Ballas, N., Yao, L., Pal, C., \& Courville, A. (2015). Delving Deeper into Convolutional Networks for Learning Video Representations, 1-11.

[41] Howard, A. G., et al. (2017). MobileNets: Efficient Convolutional Neural Networks for Mobile Vision Applications. arXiv Prepr.

[42] Simonyan, K., \& Zisserman, A. (2015). Very Deep Convolutional Networks for Large-Scale Image Recognition. The International Conference on Learning Representations 2015, 1-14. Retrieved from http://arxiv.org/abs/1409.1556

[43] Sifre, L. (2014). Rigid-Motion Scattering For Image Classification. Ecole Polytechnique.

[44] Chollet, F. (2016). Xception: Deep Learning with Depthwise Separable Convolutions. arXiv Prepr.

[45] Abadi, M., Agarwal, A., Barham, P., Brevdo, E., Chen, Z., Citro, C., ... Zheng, X. (2016). TensorFlow: Large-Scale Machine Learning on Heterogeneous Distributed Systems. arXiv Preprint. https://doi.org/10.1038/nn.3331

[46] Deng, J., Dong, W., Socher, R., Li, L., J., Li, K., \& Fei-Fei, L. (2009). ImageNet: A largescale hierarchical image database. 2009 IEEE Conf. Comput. Vis. Pattern Recognit., 248255. https://doi.org/10.1109/CVPR.2009.5206848 


\section{Authors}

Harjanto Prabowo is Rector of Bina Nusantara University, Jakarta, Indonesia and Professor of Management Information System. His research interests include knowledge management and information system.

Tjeng Wawan Cenggoro is a research assistant at Bioinformatics \& Data Science Research Center and a lecturer at School of Computer Science, Bina Nusantara University, Jakarta, Indonesia. His research focus is the application and development of Deep Learning model for agriculture and bioinformatics.

Arif Budiarto is a research assistant at Bioinformatics \& Data Science Research Center, Bina Nusantara University, Jakarta, Indonesia. His expertise areas include Internet of Things (IoT) and Human Computer Interaction (HCI).

Anzaludin Samsinga Perbangsa is a research associate at Bioinformatics \& Data Science Research Center and a lecturer at School of Information Systems, Bina Nusantara University, Jakarta, Indonesia. His research expertise is in developing tools to investigate the interplay of genetic and environmental factors in agriculture and has developed agricultural germplasm database.

Hery Harjono Muljo is a researcher at Bioinformatics \& Data Science Research Center and a lecturer at Accounting Program, Bina Nusantara University, Jakarta, Indonesia. His research expertise is in developing management information system of health institutions such as hospitals and clinics.

Bens Pardamean is Director of Bioinformatics \& Data Science Research Center and Associate Professor of Computer Science, Bina Nusantara University, Jakarta, Indonesia. His research expertise is in information technology, bioinformatics, and education, including a strong background in database systems, computer networks, and quantitative research.

Article submitted 12 March 2018. Resubmitted 30 May 2018. Final acceptance 18 July 2018. Final version published as submitted by the authors. 